OPEN ACCESS

Edited by:

Mireia Orgilés,

Miguel Hernández University of

Elche, Spain

Reviewed by:

Dunia Garrido,

University of Granada, Spain

Filippo Manti,

Sapienza University of Rome, Italy

*Correspondence:

Peter Muris

peter.muris@maastrichtuniversity.nl

Specialty section:

This article was submitted to Psychology for Clinical Settings,

a section of the journal

Frontiers in Psychology

Received: 19 February 2021

Accepted: 05 May 2021

Published: 31 May 2021

Citation:

Muris $P$, Monait N, Weijsters $L$ and Ollendick TH (2021) Symptoms of Selective Mutism in Non-clinical 3- to 6-Year-Old Children: Relations With

Social Anxiety, Autistic Features, and

Behavioral Inhibition.

Front. Psychol. 12:669907.

doi: 10.3389/fpsyg.2021.669907

\section{Symptoms of Selective Mutism in Non-clinical 3- to 6-Year-Old Children: Relations With Social Anxiety, Autistic Features, and Behavioral Inhibition}

\author{
Peter Muris ${ }^{1,2 \star}$, Nona Monait ${ }^{1}$, Lotte Weijsters ${ }^{1}$ and Thomas H. Ollendick ${ }^{3,4}$ \\ ${ }^{1}$ Department of Clinical Psychological Science, Maastricht University, Maastricht, Netherlands, ${ }^{2}$ Departement of Sielkunde, \\ Stellenbosch University, Stellenbosch, South Africa, ${ }^{3}$ Department of Psychology, Virginia Polytechnic and State University, \\ Blacksburg, VA, United States, ${ }^{4}$ Department of Psychology, Roehampton University, London, United Kingdom
}

Selective mutism (SM) is a psychiatric condition that is characterized by a failure to speak in specific social situations (e. g., at school) despite speaking normally in other situations (e.g., at home). There is abundant evidence that anxiety, and social anxiety in particular, is a prominent feature of SM, which is the main reason why this condition is currently classified as an anxiety disorder. Meanwhile, there is increasing support for the notion that autism-related problems are also involved in SM. The present study examined the relations between SM and social anxiety, autistic features, and behavioral inhibition to the unfamiliar (i.e., the tendency to react with restraint and withdrawal when confronted with unfamiliar stimuli and situations). Parents of 172 3- to 6-year-old preschool children completed an online survey for measuring the relevant constructs. Results showed that there were positive and statistically significant correlations between SM and social anxiety, autistic features, and behavioral inhibition. Regression analyses revealed that (1) both social anxiety and autistic features accounted for a significant and unique proportion of the variance in SM scores, and (2) that both of these variables no longer made a significant contribution once behavioral inhibition was added to the model. It can be concluded that while the involvement of social anxiety is unambiguous in SM, autism-related problems are also implicated. Furthermore, behavioral inhibition seems to play a key role in the non-speaking behavior of non-clinical young children.

Keywords: selective mutism, social anxiety, autism spectrum disorder, behavioral inhibition, children

\section{INTRODUCTION}

The prototypical feature of children with selective mutism (SM) is a total absence of speech in specific social situations (e.g., school) while showing a normal ability to speak in other situations [e.g., at home; American Psychiatric Association, 2013]. Thus, children with this condition often remain consistently silent in the classroom and do not respond verbally to questions and invitations of the teacher or to verbal and non-verbal communication attempts of their classmates. However, when at home with their parents, siblings, other family members, or friends, these children speak and communicate normally, expressing themselves verbally just like their peers. SM is a psychiatric disorder that usually becomes manifest during the early school years before children reach the 
age of 5 years (Steinhausen and Juzi, 1996). Children showing SM in its extreme form (i.e., <1\%; Bergman et al., 2002; Karakaya et al., 2008) are usually referred to a clinical facility for treatment because the persistent non-speaking behavior obviously hinders them in performing adequately in school and establishing friendships with other children (Manassis, 2009). It is good to bear in mind, however, that SM is a dimensional phenomenon as there are also young children who are not totally silent in certain social situations, but clearly use less spoken language as they would do in other settings (Gensthaler et al., 2020). This means that SM, just like other psychiatric conditions, is better conceptualized as a continuum rather than as a categorical diagnostic entity, and this justifies that its scientific inquiry should not only be conducted in clinical samples but also in non-clinical populations.

When looking at the origin of the selective non-speaking behavior that is so characteristic for children with SM, it is now generally assumed that social anxiety plays an important role. The scientific evidence for this notion comes from various sources. To begin with, children with SM frequently have a comorbid diagnosis of social anxiety disorder (SAD; e.g., Black and Uhde, 1995; Yeganeh et al., 2003; Vecchio and Kearney, 2005). More precisely, in a meta-analysis by Driessen et al. (2020), structured clinical interview data of 837 children with SM that were pooled from 22 studies indicated that SM was frequently accompanied by a co-occurring anxiety disorder, and in the vast majority of cases (i.e., 69\%) SAD was implicated. Furthermore, Vogel et al. (2019) conducted qualitative interviews in 65 children with SM aged 8 to 18 years to explore the content of their fears in speechrelated situations. It was found that the fears of children with SM were primarily focused on themes that are also typical for children with SAD, such as the fear of being negatively and critically evaluated by other people. Finally, comparisons of the symptom picture between children with SM and children with SAD have shown many similarities in the clinical presentation of both disorders (Manassis et al., 2003; Yeganeh et al., 2006; Gensthaler et al., 2016b; Milic et al., 2020). Most importantly, it has been noted that SM and SAD are difficult to distinguish on behavioral, psychophysiological, self-, parent-, and teacherreport measures of social anxiety (Poole et al., 2020). Given all these research findings, the current classification of SM as an anxiety disorder (see American Psychiatric Association, 2013) seems justified (Sharp et al., 2007; Viana et al., 2009; Muris and Ollendick, 2015), with some scholars even pleading for the recognition of SM as a special variant of SAD (Bögels et al., 2010).

In a recent review paper, Muris and Ollendick (2021b) suggested that-besides social anxiety—autism-related problems might also be implicated in SM. This point-of-view is controversial as autism spectrum disorder (ASD) is generally considered as an exclusion criterion for SM (American Psychiatric Association, 2013). In its purest form, the nonspeaking behavior of children with SM and children with ASD is quite different. More specifically, children with SM display the prototypical muteness when exposed to socially demanding situations, but in comfortable situations their interaction with other people is normal, with a full range of emotion and adequate social cognition (Thom et al., 2020). In contrast, children with
ASD display a more generalized impairment in social interaction: in both comfortable and uncomfortable circumstances, they exhibit impediments in social emotion, cognition, skills, and motivation (Pallathra et al., 2018), that may sometimes be expressed in non-speaking behavior. However, as noted above, contemporary psychiatry views mental disorders as dimensions with low symptom levels on the one hand and high symptom levels on the other hand (Krueger and Piasecki, 2002; Hudziak et al., 2007), which implies that relations among disorders and overlap in symptoms are the rule rather than the exception (Plana-Ripoll et al., 2019).

Indeed, there is increasing evidence showing that SM and ASD are related psychopathological conditions and that it is difficult to maintain an absolute diagnostic boundary between both disorders. For instance, in a study by Steffenburg et al. (2018), the medical records of 97 children with SM were subjected to a systematic analysis to establish the possible presence of ASD. The results showed that no less than $63 \%$ of the children with SM also fulfilled the diagnostic criteria for ASD, while an additional $20 \%$ of the children with SM displayed autistic features, hence showing subclinical signs of this neurodevelopmental disorder. Although the Steffenburg et al. (2018) study has some methodological shortcomings (e.g., reliance on retrospective chart reviews), findings do suggest that there is considerable co-occurrence of SM and ASD. In a further investigation, Klein et al. (2019) administered a standardized parent- and teacher-report scale to assess psychopathological symptoms in 42 children with SM aged between 2 and 14 years. The scale also contained a screen for ASD and it was found that $80 \%$ of the children with SM scored above the cut-off on this autism probability index, indicating that many of them showed clinical signs of social and communication problems and stereotyped interests and behaviors. In addition, Cholemkery et al. (2014) asked parents of 6- to 18-year-old children with SM, social anxiety disorder, and ASD as well as typically developing children to complete a standardized scale measuring autistic symptoms in five domains, namely social awareness, social cognition, social communication, social motivations, and repetitive/restricted behaviors. The results showed that all children with a clinical diagnosis displayed higher levels of autistic symptoms than the typically developing children. Children with ASD clearly displayed the highest levels of social interaction impairments, but on two domains (i.e., social communication and social motivation) children with SM also exhibited elevated scores (as compared to children with social anxiety disorder), which implies that they were also relatively high on the autism spectrum. Other research has indicated that children with SM appear to display a similar cognitive deficit (i.e., impairments in initiating joint attention; Nowakowski et al., 2011) and share a common genetic liability (i.e., a specific polymorphism in the contactin-associated protein-like 2 gen; Stein et al., 2011) as young people with ASD.

In view of this increasing evidence, Muris and Ollendick (2021b) argued that the presence of ASD (or at least autistic traits) likely increases children's proneness to develop SM. In specific, the social skills and social cognition deficits associated with this neurodevelopmental problem might fuel social anxiety 
symptoms as well as prompt muteness as an avoidance strategy to deal with the excessive symptomatology elicited by specific social situations. Further, the rigidity and cognitive inflexibility of children with ASD will enhance social difficulties thereby further intensifying the social anxiety, but also promoting the persistent non-speaking behavior displayed by children with SM. It is important to note that in most of children, $\mathrm{SM}$ is primarily an anxiety-driven condition (Cohan et al., 2008; e.g., Capozzi et al., 2018). However, various scholars have noted out that SM is a heterogeneous disorder (e.g., (Mulligan, 2012)) and that there are children in which-besides (social) anxiety-other problems such as developmental delay (Kristensen, 2000), language expression difficulties (Manassis et al., 2007), oppositional behavior (Diliberto and Kearney, 2018), and presumably ASD or related features are implicated as well.

Temperament might be another factor contributing to SM. Of special interest is the temperament typology of "behavioral inhibition to the unfamiliar" (BIU; Kagan, 1997), which can be defined as a predisposition characterized by restraint in engaging with the external world combined with a tendency to search the environment for potential threats and to avoid or withdraw from unfamiliar people and situations. A host of studies have established that BIU is an important risk factor for SAD (Clauss and Blackford, 2012), but given the fact that reduced speech in social situations is one of the defining features of an inhibited temperament (e.g., Garcia Coll et al., 1984; Van Brakel et al., 2004), it is obvious to also explore its link with SM. In a first study of this topic, Gensthaler et al. (2016a) employed a retrospective parent-rating scale to measure inhibited temperament features in 3- to 18-year-old children with $\mathrm{SM}, \mathrm{SAD}$, other internalizing problems, and healthy controls. It was found that children with SM and SAD were reported to have been more inhibited during their early childhood years than children with other internalizing behaviors and healthy controls. In general, the levels of BIU of children with SM and ASD were rated as comparably high, although on the specific domain of shyness children with SM even displayed higher levels of inhibition than their counterparts with SAD. Further research by Milic et al. (2020) relied on a cross-sectional, multi-method research design to compare BIU features among children with SM, children with SAD, and nonclinical controls. Parent ratings revealed that children with SM and children with SAD both had a greater tendency to withdraw from novel situations and unfamiliar people than the non-clinical control children. Observations conducted during a series of performance tasks indicated that children with SM scored higher on a few measures of inhibition (i.e., latency to initiate gestures, latency to initiate speech, total amount of speech) than children with SAD. In a final investigation by Muris et al. (2016), 57 non-clinical children aged 3 to 6 years performed two speech tasks to assess the number of spoken words, while their parents completed a set of questionnaires for measuring children's levels of SM, social anxiety, and an inhibited temperament. Significant associations were noted among all variables, but the correlation between BIU and SM symptoms was particularly robust, and it was also found that this temperament typology was the best predictor of the number of spoken words during the standardized speech tasks. Taken together, the available evidence demonstrates that BI, which already has been established as an important risk factor for SAD, is also clearly implicated in SM.

The observation that SM is associated with multiple factors fits nicely within a developmental psychopathology framework (Cicchetti and Cohen, 2006). That is, the selective non-speaking behavior of children with SM does not seem to develop as the result of one deterministic variable, but likely originates from a complex of vulnerability factors that jointly increase the probability (risk) for this psychiatric condition to occur (Cohan et al., 2006; Viana et al., 2009; Muris and Ollendick, 2015). In keeping with the principle of equifinality (i.e., any one outcome might result from multiple and diverse pathways; see Cicchetti and Rogosch, 1996), the exact constellation of vulnerability factors can be and most likely is different across children. However, it is important to study the relative contributions of various risks on a group level as such information can be highly relevant for giving direction to the clinical management of young people with a given disorder.

So far, the research on etiological models of SM has primarily examined vulnerability factors in isolation. The evidence suggests that social anxiety plays a dominant role in the origins of this disorder, but other variables such as autistic features and a behaviorally inhibited temperament also seem to be implicated. Meanwhile, we know little about the unique contributions of each of these variables to SM. This seems all the more important when acknowledging that there appears to be considerable overlap among these vulnerability factors. More precisely, SAD and ASD appear to be closely related (Spain et al., 2018) and the same is true for BIU and SAD (Ollendick and Hirshfeld-Becker, 2002; Clauss and Blackford, 2012). So far, little is known about the link between BIU and ASD, although it should be noted that children with autism-related problems often display reticence and distress when meeting unfamiliar people or facing novel situations, which is also typical for temperamental inhibition (Ersoy, 2019).

With these issues in mind, the present study made a first attempt to examine the (unique) relations between social anxiety, autistic features, and BIU on the one hand and symptoms of SM on the other hand. For this purpose, the parents of 172 non-clinical children aged 3 to 6 years completed a survey containing the Selective Mutism Questionnaire (SMQ; Bergman et al., 2008), the social anxiety subscale of the Preschool Anxiety Scale-Revised (Edwards et al., 2010), the Autism Spectrum Questionnaire (ASQ; Van der Ploeg and Scholte, 2014), and the Behavioral Inhibition Questionnaire-Short Form (BIQ-SF; Edwards, 2007). It was hypothesized that there would be positive correlations between SM and the other constructs. The most substantial associations were expected to be found between $\mathrm{SM}$ symptoms and social anxiety/BIU, whereas the relation between SM symptoms and autistic features was expected to be considerably smaller. Furthermore, based on theoretical notions (Muris and Ollendick, in press), it was hypothesized that even when controlling for social anxiety, autistic features will still make a unique contribution to SM symptoms. The role of BIU was investigated more exploratively, but on the basis of an earlier study (Muris et al., 2016) it can be expected that this temperament typology makes a significant contribution to symptoms of SM even when controlling for 
its shared variance with the other constructs and social anxiety in particular.

\section{METHOD}

\section{Participants and Procedure}

Participants in this study were the parents of 172 non-clinical children ( 96 boys and 76 girls) aged $3(n=45,26.2 \%), 4(n=61$, $35.5 \%), 5(n=42,24.4 \%)$, or $6(n=24,14.0 \%)$ years; the mean age was 4.26 years $(S D=1.00)$. The sample was recruited via 3 daycare facilities and two elementary schools in the Southern part of The Netherlands, as well as by means of a snowball sampling method (Goodman, 1961) starting with the acquaintances of the second and third author using online social media platform. To be included in the study, participants needed to be the parent of a child in the preschool age range ( 3 to 6 years) and to possess sufficient command of the Dutch language in order to be able to complete the questions of the survey. There were no exclusion criteria for this study.

In most cases, the mothers completed the survey $(n=152$, $88.3 \%)$. All families had a Caucasian background and the vast majority of the parents and children ( $n=169,98.2 \%)$ were of Dutch nationality; only some families included members with South European or Middle Eastern roots. The language spoken at home was mainly Dutch $(n=146,84.9 \%)$ or the Dutch dialect that is typically spoken in this part of The Netherlands $(n=24$, $13.9 \%)$; a foreign language (Italian and Greek) was the dominant language in only two families (1.2\%).

Parents first received an information letter describing the purpose of this study and an informed consent form. After signing the informed consent form, they were sent a link guiding them to the online survey. Following this, parents completed the set of questionnaires describing their child's behaviors in relation to the relevant constructs. After finishing the questionnaires, the parents were given the opportunity to share their email address in case they wished to receive information on the results of the study. The study was approved by the Ethical Review Committee of Psychology and Neuroscience at Maastricht University (reference number: ERCPN-221_50_03_2020).

\section{Assessment}

Symptoms of SM were measured with the SMQ (Bergman et al., 2008), which is a 17 -item parent-rating scale measuring the frequency of non-speaking behavior in three settings where children are normally expected to speak: at school (e.g., "When appropriate, my child speaks in groups or in front of the class"), at home/with family (e.g., "When appropriate, my child speaks with family friends who are well-known to him/her"), and other social situations (e.g., "When appropriate, my child speaks to store clerks and/or waiters"). Items were rated on a 4-point Likert scale, with $0=$ never, $1=$ seldom, $2=$ often, and $3=$ always. A total score (range $0-51$ ) can be calculated by summing ratings across all items. Lower scores on the SMQ indicate a lower frequency of speaking behavior and thus higher levels of SM. To enhance interpretability, the main analyses were conducted using a reversed SMQ total score for which higher scores reflect higher symptom levels of SM. Previous studies have shown that the SMQ is a reliable scale (with Cronbach's in the 0.80 to 0.90 range) that relates in a theoretical meaningful way with other measures (Bergman et al., 2008; Letamendi et al., 2008), predicts the diagnostic status of SM (Oerbeck et al., 2020), and is sensitive to document treatment effects (Bergman et al., 2013; Oerbeck et al., 2015).

A subscale of the PAS-R (Edwards et al., 2010) was used to measure children's level of social anxiety. The PAS-R is a 30item adaptation of the Preschool Anxiety Scale (Spence et al., 2001), a parent-report questionnaire that assesses symptoms of anxiety disorders in young children. The social anxiety subscale consists of 6 items such as "My child worries that he/she will do something to look stupid in front of other people," and "My child is afraid to go up to a group of children to join their activities." Items are scored on a 4 -point Likert scale ranging $0=$ not at all true to $4=$ very often true. A total social anxiety score (range 0-28) can be computed, with higher scores indicating higher levels of social anxiety symptomatology. In general, the PAS$\mathrm{R}$ has been shown to be a reliable and valid index of anxiety in children of a preschool age. The internal consistency and test-retest reliability estimates of the social anxiety scale are in the 0.70 to 0.80 range, its scores are predictive of a clinical diagnosis of SAD, and correlate significantly and robustly with other measures of anxiety and emotional symptoms (Edwards et al., 2010; Stuijfzand and Dodd, 2017; Orgiles et al., 2018; Gudmundsdottir et al., 2019).

The ASQ (Van der Ploeg and Scholte, 2014) evaluates the presence of symptoms of ASD in children. The questionnaire consists of 24 items that can be allocated to two subscales: (1) Interactive and communicative problems (all reversed items, e.g., "My child actively seeks contact with other children," "My child gets along with different kinds of people"), which covers the persistent social interaction and social communication impairments displayed by young people with this neurodevelopmental condition, and (2) Odd, deviant behaviors (e.g., "My child shows strange, repetitive behaviors," "My child has difficulties when he/she has to switch from one task to another"), which pertains to the restricted repetitive behaviors and interests of children with ASD. Parents rate the applicability of each item for their child using a 5-point Likerttype scale ranging from $1=$ not at all to $5=$ very much. Scores can be computed for the full scale as well as for the two subscales by summing the ratings across relevant items. The ratings on items referring to Interactive and communicative problems are recoded, so that in all cases higher scores reflect higher levels of ASD symptomatology. Psychometric evaluation of the ASQ (Van der Ploeg and Scholte, 2014) has indicated that the scale is reliable in terms of internal consistency (with Cronbach's alphas ranging between 0.91 and 0.94 for nonclinical children and between 0.84 and 0.90 for children with ASD) and test-retest stability (with intraclass correlations over a 4 -week period varying between 0.84 and 0.91 ) as well as interrater agreement (intraclass correlations between 0.62 and 0.82). Further, scores on the ASQ discriminate well between children with and without ASD and correlate positively and substantially with an alternative measure of symptoms of this neurodevelopmental disorder. 
The BIQ-SF (Edwards, 2007) is the short version of the Behavioral Inhibition Questionnaire (Bishop et al., 2003). This parent-report scale contains 14 items measuring features of the temperament typology of BIU in children. Representative items include "My child gets upset when being left in new situations for the first time, for example kindergarten" and "My child approaches new situations or activities very hesitantly," which parents rate on a 6 -point Likert scale, ranging from $1=$ hardly ever to $6=$ almost always. A total score can be computed by summing ratings across all items, with a higher score being indicative for a higher level of BIU. The internal consistency of the BIQ-SF was demonstrated to be good (with a Cronbach's alpha of 0.92 for the total score) and scores on the scale were found to be fairly stable over a period of 1 to 2 years (test-retest correlations being 0.73 and 0.65 , respectively; Vreeke et al., 2012). In addition, support was obtained for the validity of the BIQ-SF as scores correlated positively and significantly with behavioral observations of young children's inhibited temperament (Bishop et al., 2003; Vreeke et al., 2012).

\section{Data Analyses}

Data were analyzed using the Statistical Package for Social Sciences (SPSS Version 25). First, descriptive statistics (mean scores, standard deviations, reliability coefficients) were calculated, and gender differences and age effects were investigated by means of independent $t$-tests and correlations, respectively. Second, Pearson correlations were computed to examine the relations between symptoms of SM (SMQ) on the one hand and social anxiety (PAS-R), autistic features (ASQ), and BIU (BIQ-SF) on the other hand. Third, to explore unique contributions of various constructs to symptoms of SM, linear regression analyses were conducted with the SMQ total score was the dependent variables and other constructs served as the predictors. In specific, three models were tested (see Figure 1). In a first model, it was explored whether social anxiety (PAS-R) and autistic features (ASQ total score) each explain a unique proportion of the variance in selective mutism symptoms (SMQ), which would be in line with notion of Muris and Ollendick (in press). The second model was basically a refinement of the first model. Apart from the social anxiety (PAS$\mathrm{R})$, the two subscales of the ASQ were included separately in the regression equation in order to find out the relative contributions of Interactive/communicative problems and odd/deviant behaviors. The third and final model not only included social anxiety and autistic features but also incorporated behavioral inhibition as predictor, and hence will give insight on the relative contributions of psychopathology indicators and temperament characteristics to SM symptoms in non-clinical children.

\section{RESULTS}

\section{General Findings}

Before addressing the main results of the present study, a number of general findings are reported. First, a comparison of the SMQ, PAS-R, ASQ, and BIQ-SF scores with normative data of these measures (Spence et al., 2001; Vreeke et al., 2012; Van der Ploeg and Scholte, 2014; Oerbeck et al., 2020) revealed that parents

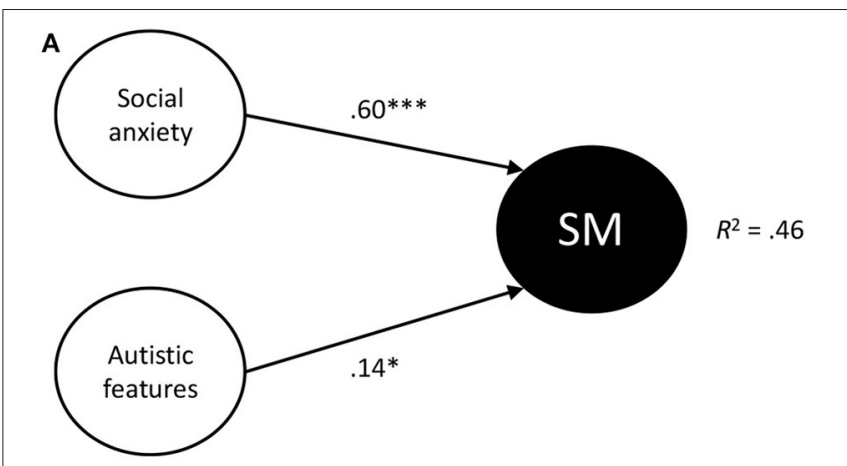

B

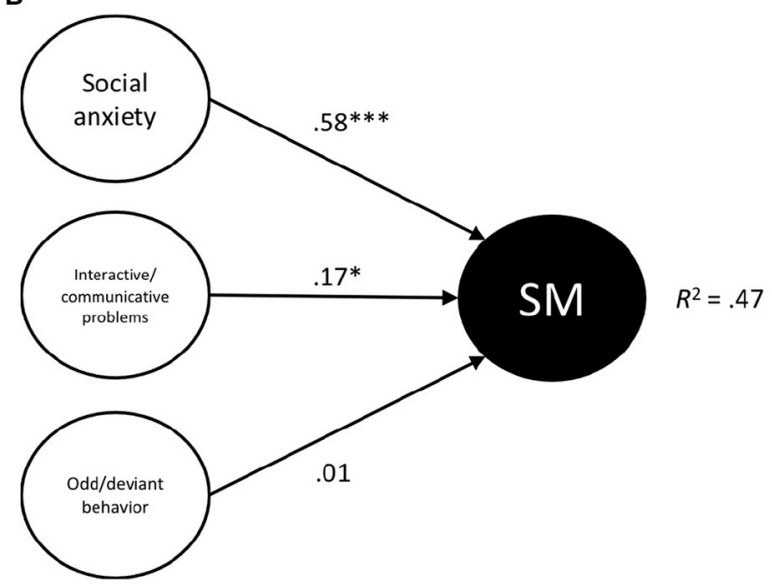

c

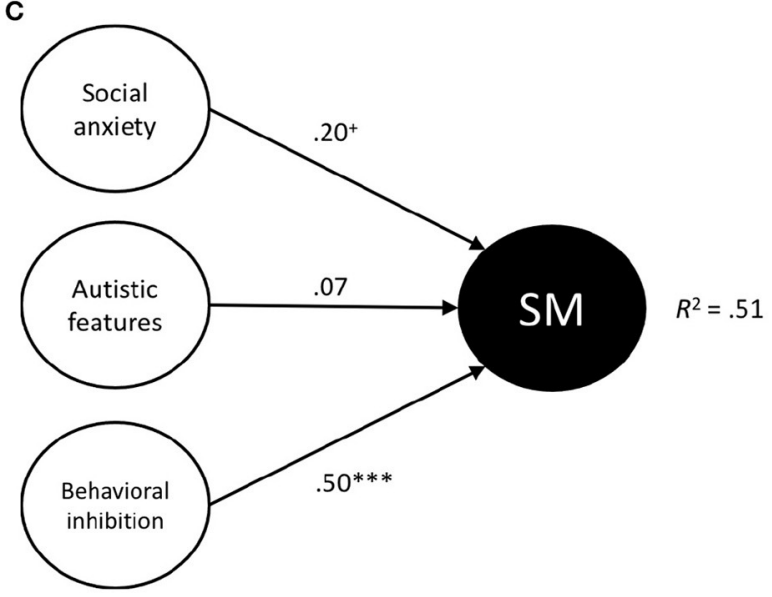

FIGURE 1 | (A-C) Schematic representation of the three regression models that were tested. Standardized betas and $R^{2}$ values are shown. SM, Symptoms of Selective Mutism. ${ }^{+} p<0.10,{ }^{*} p<0.05,{ }^{\star \star \star} p<0.001$

rated the children in the present study as clearly falling in the normal range of selective mutism, social anxiety, autistic features, and behavioral inhibition. Second, demographic variables did not have a significant influence on the constructs that were assessed in this study. That is, no gender differences were found for any of the measures (all $t$ 's $\leq 1.54, p$ 's $\geq 0.11$ ), implying that boys and girls were rated as displaying comparable levels 
TABLE 1 | Mean scores (standard deviations) and reliability coefficients for parent-report questionnaires of children's symptoms and temperament features as well as Pearson correlations among these measures.

\begin{tabular}{|c|c|c|c|c|c|c|c|}
\hline & $M(S D)$ & Cronbach's $\alpha$ & 1 & 2 & 3 & 4 & 5 \\
\hline 1. SMQ Selective mutism & $38.36(10.07)^{\dagger}$ & 0.93 & & & & & \\
\hline 2. PAS-R Social anxiety & $9.51(5.62)$ & 0.90 & $0.67^{\star \star \star}$ & & & & \\
\hline 3. BIQ-SF Behavioral inhibition & $41.36(14.64)$ & 0.94 & $0.71^{\star \star \star}$ & $0.88^{\star \star \star}$ & & & \\
\hline 4. ASQ Autistic features & $52.72(11.56)$ & 0.89 & $0.43^{\star * \star}$ & $0.48^{\star \star *}$ & $0.54^{\star \star \star}$ & & \\
\hline 5. ASQ Interactive/communicative problems & $26.61(6.83)$ & 0.87 & $0.47^{\star \star \star}$ & $0.52^{\star \star \star}$ & $0.60^{\star \star \star}$ & $0.89^{\star \star \star}$ & \\
\hline 6. ASQ Odd/deviant behaviors & $26.11(6.27)$ & 0.81 & $0.28^{\star \star \star}$ & $0.31^{\star \star \star}$ & $0.33^{\star \star \star}$ & $0.87^{\star \star \star}$ & $0.56^{\star \star \star}$ \\
\hline
\end{tabular}

N = 172. SMQ, Selective Mutism Questionnaire; PAS-R, Preschool Anxiety Scale-Revised; BIQ-SF, Behavioral Inhibition Questionnaire-Short Form; ASQ, Autism Spectrum Questionnaire.

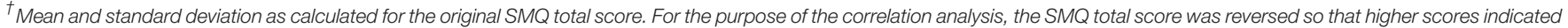
higher levels of SM symptoms. ${ }^{* \star} p<0.001$.

of psychopathology and temperament. Further, no significant relationships were noted between age and the assessed constructs ( $r$ 's between -0.08 and $0.11, p$ 's $\geq 0.16$ ), which was not that surprising given that the age range of the children included in the present study was quite small (i.e., 3 to 6 years). Third and finally, all questionnaires showed good to excellent internal consistency reliability, with Cronbach's alpha coefficients varying between 0.81 and 0.94 (see Table 1).

\section{Correlations Between SM, Social Anxiety, Autistic Features, and BIU}

Correlations between SM symptoms and symptoms of social anxiety and ASD as well as features of the temperamental construct BIU are shown in Table 1. Three main conclusions can be derived from this table. First, a robust positive and substantially significant correlation was found between the SMQ and the PAS-R social anxiety subscale $(r=0.67)$ : as hypothesized, higher levels of SM symptoms were accompanied by higher levels of social anxiety. Second, substantial positive and statistically significant correlations were also noted between BIQ-SF scores on the one hand and SMQ and PAS-R social anxiety scores on the other hand ( $r$ 's being 0.71 and 0.88 , respectively), which means that higher levels of the temperament characteristic of BIU were associated with higher levels of both SM and social anxiety. Third, SMQ scores were also positively correlated with ASQ scores $(r$ $=0.43$ ), which means that symptoms of SM were associated with higher levels of autistic features. A test for comparing correlated correlation coefficients indicated, as predicted, that the correlation between SM and social anxiety was significantly stronger than the correlation between SM and ASD features $(Z=$ 3.97, $p<0.001$ ).

An additional finding that emerged from the correlational analysis was that SMQ scores were statistically significantly correlated with both the interactive/communicative problems and odd/deviant behaviors subscales of the ASQ [ $r$ 's being 0.47 and 0.28 , with the former correlation being significantly stronger than the latter correlation $(Z=2.91, p<0.01)]$.

Further, a statistically significant positive correlation was found between PAS-R social anxiety and ASQ scores ( $r$ 's between 0.31 and 0.52 ), which indicates that higher levels of social anxiety symptoms were accompanied by higher levels of autistic features. A final result concerned the positive and statistically significant correlations between BIQ-SF and ASQ scores: notably, higher levels of BIU were associated with higher levels of autistic features $(r=0.54)$ and this appeared true for interactive/communicative problems $(r=0.60)$ as well as odd/deviant behaviors $(r=0.33)$.

\section{Unique Contributions of Various Constructs to Symptoms of SM}

To examine the unique contributions of various constructs to symptoms of SM, three linear regression analyses with SMQ scores as the dependent variable were conducted (see above: section Data Analyses). As demographic did not have a large impact on the variables included in this study, we did not include them as predictors in the regression models. However, running the analyses with gender and age as additional predictors yielded highly similar results. Before discussing the results of the regression analysis, two general remarks should be made. First, diagnostic tests were conducted to detect multicollinearity issues. Results showed that Variance Inflation Factor values were all $\leq 4.84$, while Tolerance values were $\geq 0.22$, which points out that there were no substantial violations of multicollinearity (O'Brien, 2007). Second, it was found that each of the tested models were statistically significant (all $F$ 's $\geq 49.00, p$ 's $<0.001$ ) and that across various analyses predictor variables explained between 46 and $51 \%$ of the total variance in symptoms of SM.

The main results of the three regression analyses are shown in Table 2. The first regression analysis with the PAS-R social anxiety score and ASQ total score as the predictors revealed that both social anxiety $(\beta=0.60, t=9.32, p=0.000)$ and autistic features $(\beta=0.14, t=2.22, p=0.028)$ made a positive, statistically significant, and independent contribution to symptoms of SM. The second analysis in which PAS-R social anxiety and ASQ subscales were the predictor variables confirmed the unique contribution of social anxiety $(\beta=0.58$, $t=8.76, p=0.000$ ) and also showed that the effect of autistic features was mainly carried by the interactive/communicative difficulties associated with this neurodevelopmental condition $(\beta=0.17, t=2.18, p=0.031)$. No statistically significant effect of odd/deviant behaviors was found $(\beta=0.01, t=0.09$, $p=0.930)$. The third and final regression analysis included PAS-R social anxiety, the ASQ total score, and BIQ-SF as predictors, and found that only the temperament trait of BIU made a unique and statistically significant contribution to 
TABLE 2 | Main results of the regression analyses in which symptoms of SM were predicted from social anxiety, autistic features, and behavioral inhibition.

\begin{tabular}{|c|c|c|c|c|c|}
\hline & B & $95 \% \mathrm{Cl}$ & $S E$ & $\beta$ & $R^{2}$ \\
\hline $\mathrm{SMQ}$ Selective mutism ${ }^{\dagger}$ & & & & & $0.46^{\star \star \star}$ \\
\hline PAS-R Social anxiety & 1.07 & {$[0.85,1.30]$} & 0.12 & $0.60^{\star \star \star}$ & \\
\hline ASQ Autistic features & 0.12 & {$[0.01,0.24]$} & 0.06 & $0.14^{\star}$ & \\
\hline SMQ Selective mutism & & & & & $0.47^{\star \star \star}$ \\
\hline PAS-R Social anxiety & 1.04 & {$[0.80,1.27]$} & 0.12 & $0.58^{\star \star \star}$ & \\
\hline ASQ Interactive/communicative problems & 0.24 & {$[0.02,0.46]$} & 0.11 & $0.17^{\star}$ & \\
\hline ASQ Odd/deviant behaviors & 0.01 & {$[-0.21,0.23]$} & 0.11 & 0.01 & \\
\hline SMQ Selective mutism & & & & & $0.51^{\star \star \star}$ \\
\hline PAS-R Social anxiety & 0.35 & {$[-0.05,0.75]$} & 0.20 & $0.20^{+}$ & \\
\hline ASQ Autistic features & 0.06 & {$[-0.05,0.17]$} & 0.06 & 0.07 & \\
\hline BIQ-SF Behavioral inhibition & 0.34 & {$[0.18,0.50]$} & 0.08 & $0.50^{\star \star \star}$ & \\
\hline
\end{tabular}

$N=172$. SMQ, Selective Mutism Questionnaire; PAS-R, Preschool Anxiety Scale-Revised; BIQ-SF, Behavioral Inhibition Questionnaire-Short Form; ASQ, Autism Spectrum Questionnaire.

${ }^{\dagger}$ The SMQ total score was reversed so that higher scores indicated higher levels of SM symptoms. ${ }^{+} p<0.10,{ }^{*} p<0.05,{ }^{\star \star *} p<0.001$.

symptoms of $\mathrm{SM}(\beta=0.50, t=4.23, p=0.000)$. In this model, the contribution of social anxiety was also positive but only marginally significant $(\beta=0.20, t=1.73, p=0.086$ ), whereas ASD symptoms did no longer explain a significant proportion of the variance in SM symptomatology $(\beta=0.07$, $t=1.09, p=0.278)$.

\section{DISCUSSION}

The present study examined psychopathological and temperamental correlates of SM symptoms in a non-clinical sample of 3- to 6-year-old children by means of a parent survey. The results revealed there was a robust and statistically significant correlation between social anxiety and SM symptoms. This is in line with previous clinical studies showing that the comorbidity between SAD and SM is high (Driessen et al., 2020) and that there are clear similarities between both disorders in terms of fear content (Vogel et al., 2019) and clinical presentation (Manassis et al., 2003; Yeganeh et al., 2006; Gensthaler et al., 2016b; Milic et al., 2020; Poole et al., 2020). But even in nonclinical research, the substantial correlation between symptoms of social anxiety and SM has been documented (Muris et al., 2016). On the basis of the intimate link between both conditions it has been argued that SM can best be viewed as a special variant of SAD. Some advocates of this notion have suggested that SM should be regarded as a more extreme variant of SAD (e.g., Black and Uhde, 1992), while others have put forward that SM can best be viewed as an early developmental manifestation of SAD (e.g., Bergman et al., 2002). Importantly, the robust association with social anxiety justifies the position of SM among the anxiety disorders, which has also implications for the clinical management of the disorder (Muris and Ollendick, 2021a). More specifically, clinicians should use instruments to assess the level of social anxiety associated with this condition and apply cognitive-behavioral interventions to treat the fear-driven non-speaking behavior of children with this condition (Bergman et al., 2013; Oerbeck et al., 2014; Cornacchio et al., 2019).
Furthermore, it was found that autistic features were also positively associated with symptoms of SM. When controlling for concurrent levels of social anxiety the relation between ASD and SM symptoms clearly attenuated but still remained positive and statistically significant. This corroborates results obtained in previous clinical research showing that a substantial proportion of the children with SM display autistic features (Steffenburg et al., 2018; Klein et al., 2019) as well as cognitive or pathophysiological features associated with this neurodevelopmental disorder (Nowakowski et al., 2011; Stein et al., 2011). This result also provides further support for the model recently described by Muris and Ollendick (2021b) in which ASD-related problems are proposed as one of the psychopathological phenomena contributing to the persistent non-speaking behavior of children with SM. These authors assume that both symptom clusters of ASD, namely (a) social communication and interaction difficulties, and (b) restricted and repetitive behaviors and interests, each make an independent contribution to SM. The present results indicate that although both ASD symptoms clusters were significantly and positively correlated with symptoms of SM, only the communication and interaction difficulties (as measured by ASQ Interactive/communicative problems) made a unique statistically significant contribution. The most plausible explanation for this finding is that communication and interaction deficiencies are directly relevant for children's social functioning (Pallathra et al., 2018) and thus exert their influence even when symptom levels are relatively low. Meanwhile, repetitive and restrictive behaviors and interests are not necessarily social in nature and hence may need to be more intense and severe before they start to have an impact on children's (speaking) behavior in social situations. According to normative data of the ASQ (Van der Ploeg and Scholte, 2014), scores on both the "interactive/communicative problems" and "odd/deviant behavior" subscales were rather low in this non-clinical population (i.e., mean scores fell in the lowest decile of children with ASD). Thus, it seems important to test the relative contributions of both symptoms clusters to $\mathrm{SM}$ in a sample of clinically referred children who will not only 
show higher levels but also more variation in the prototypical symptoms of this neurodevelopmental disorder, which could result in finding that repetitive and restrictive behaviors and interests also play a role in SM (see e.g., Magiati et al., 2016; Teh et al., 2017).

This study also showed that there is a strong relationship between SM and BIU, which is in agreement with the results of previous research (Gensthaler et al., 2016a; Muris et al., 2016). In the present study, not only a robust positive correlation between SM symptoms and features of this temperament characteristic was found, but the results of the regression analysis also demonstrated that even when controlling for symptoms of social anxiety and ASD, BIU still made a significant and unique contribution to SM symptoms. In fact, BIU emerged as the only statistically significant predictor variable, while social anxiety and autistic features no longer explained a significant proportion of the variance once this temperament factor was added to the regression model. Again, this may well have to do with the non-clinical sample that was investigated in this study, in which symptom levels of social anxiety, autistic features, and SM in general were quite low. The fact that BIU appeared to display a unique relation with SM aligns with the notion of Perez-Edgar and Guyer (2014) that this temperament characteristic can be seen as a prodrome of anxiety pathology which can be easily and reliably detected in non-clinical populations.

An additional finding of the current investigation concerned the positive relation between BIU and autistic features. Surprisingly, few studies have directly examined this link, although the key features of BIU share similarities with the typical clinical symptoms of "insistence on sameness" and "reactions of distress to small changes" displayed by many children with ASD (American Psychiatric Association, 2013). One exception is a recent investigation by Esroy et al. (2020) who found some evidence that children at high risk for ASD displayed higher levels of BIU than children who were at low risk for this neurodevelopmental disorder. Meanwhile, there is also research indicating that temperament and personality features related to BIU such as high emotional instability (neuroticism), low sociability (extraversion), and low effortful control (see Muris and Dietvorst, 2006) are more clearly present in children with ASD than in typically developing children (e.g., Samyn et al., 2011; Macari et al., 2017; Lodi-Smith et al., 2019).

It needs to be acknowledged that the present investigation suffers from a number of limitations. First, as already mentioned, the study relied on a non-clinical sample of children displaying relatively low symptom levels of SM and other psychopathologies who were not subjected to a formal psychiatric evaluation. So, replication of this research in clinically referred children or non-clinical children who are carefully assessed for psychiatric disorders seems very important to gain more insight on the relations between SM on the one hand and social anxiety, autistic features, and BIU on the other hand. Second, this study solely relied on parent-report questionnaires to measure symptoms of SM and the other constructs. Because all scales included items that were concerned with the assessment of social difficulties, the data were particularly prone to the commonmethod variance bias. Given the young age of the children that were included in this study, the use of child self-reports was not feasible, but obviously observation-based procedures as well as the employment of scales to be completed by day care facility workers and teachers would have provided important crossvalidational information. Third, an important part of the data was collected via snowball sampling, a method that was used because due the Covid-19 pandemic schools and daycare facilities were either closed or less willing to participate in research because they were already overloaded by handling other logistic issues. However, a disadvantage of the snowball sampling method is that one does not know to what extent results are generalizable to the whole population (Balter and Brunet, 2012). Fourth, although this investigation focused on a number of relevant psychopathological and temperamental correlates of SM, it is good to keep in mind that other factors have also been connected to this condition. Prominent examples are developmental delays (Kristensen, 2000), speech and language problems (Manassis et al., 2007), and externalizing symptoms (Diliberto and Kearney, 2018). Thus, in order to get a complete picture of possible antecedents of children's non-speaking behavior, it will be necessary to also include scales or instruments that assess these constructs. Fifth and finally, the study was correlational in nature, which means that although the main analyses were conducted with symptoms of SM as the to be explained (i.e., dependent) variable, in actuality no conclusions can be drawn in terms of cause-effect relationships. Thus, prospective, longitudinal research in which symptoms of SM, social anxiety, ASD and temperamental inhibition are repeatedly assessed over the course of the preschool and early primary school years could provide important information on the temporal associations among these constructs and the psychopathological and temporal antecedents of SM in children.

In spite of these shortcomings, the current findings indicate that non-speaking behavior in children is positively associated with social anxiety, autistic features, and the temperament characteristic of BIU, and thus support theoretical notions on the multifactorial origins of SM (Cohan et al., 2006; Viana et al., 2009; Muris and Ollendick, 2015, 2021b). In this sample of nonclinical children, BIU appeared to be the best "predictor" of SM symptoms, which suggests that this temperament trait might be a particularly important target for prevention strategies. In this light, it is good to note that Rapee and Edwards (2009) have developed a parent-based intervention programs by means of which inhibited behaviors in children can be effectively reduced. The program has already been shown to be effective in reducing the development of common childhood anxiety disorders such as separation anxiety disorder and social phobia (Rapee et al., 2010), and so it would be of interest to determine if this approach would also be successful in preventing the development of SM.

\section{DATA AVAILABILITY STATEMENT}

The raw data supporting the conclusions of this article will be made available by the authors, without undue reservation.

\section{ETHICS STATEMENT}

The studies involving human participants were reviewed and approved by Ethical Research Committee of Psychology and 
Neuroscience, Maastricht University. Written informed consent to participate in this study was provided by the participants' legal guardian.

\section{AUTHOR CONTRIBUTIONS}

PM designed the study, supervised the data collection and processing, conducted the statistical analyses,

\section{REFERENCES}

American Psychiatric Association (2013). Diagnostic and Statistical Manual of Mental Disorders, 5th Edn. Arlington, VA: American Psychiatric Association.

Balter, F., and Brunet, I. (2012). Social research 2.0: virtual snowball sampling method using Facebook. Intern. Res. 22, 55-74. doi: 10.1108/10662241211199960

Bergman, R. L., Gonzalez, A., Piacentini, J., and Keller, M. L. (2013). Integrated behavior therapy for selective mutism. Behav. Res. Ther. 51, 680-689. doi: 10.1016/j.brat.2013.07.003

Bergman, R. L., Keller, M. L., Piacentini, J., and Bergman, A. J. (2008). The development and psychometric properties of the Selective Mutism Questionnaire. J. Clin. Child Adolesc. Psychol. 37, 456-464. doi: 10.1080/15374410801955805

Bergman, R. L., Piacentini, J., and McCracken, J. T. (2002). Prevalence and description of selective mutism in a school-based sample. J. Am. Acad. Child Adolesc. Psychiatry 41, 938-946. doi: 10.1097/00004583-200208000-00012

Bishop, G., Spence, S. H., and McDonald, C. (2003). Can parents and teachers provide a reliable and valid report of behavioral inhibition? Child Dev. 74, 1899-1917. doi: 10.1046/j.1467-8624.2003.00645.x

Black, B., and Uhde, T. W. (1992). Case study: elective mutism as a variant of social phobia. J. Am. Acad. Child Adolesc. Psychiatry 31, 1090-1094. doi: 10.1097/00004583-199211000-00015

Black, B., and Uhde, T. W. (1995). Psychiatric characteristics of children with selective mutism: a pilot study. J. Am. Acad. Child Adolesc. Psychiatry 34, 847-856

Bögels, S. M., Alden, L., Beidel, D. C., Clark, L. A., Pine, D. S., Stein, M. B., et al. (2010). Social anxiety disorder: questions and answers for the DSM-V. Depress. Anxiety 27, 168-189. doi: 10.1002/da.20670

Capozzi, F., Manti, F., Di Trani, M., Romani, M., Vigliante, M., and Sogos, C. (2018). Children's and parents' psychological profiles in selective mutism and generalized anxiety disorder: a clinical study. Eur. Child Adolesc. Psychiatry 27, 775-783. doi: 10.1007/s00787-017-1075-y

Cholemkery, H., Mojica, L., Rohrmann, S., Gensthaler, A., and Freitag, C. M. (2014). Can autism spectrum disorders and social anxiety disorder be differentiated by the Social Responsiveness Scale in children and adolescents. J. Autism Dev. Disord. 44, 1168-1182. doi: 10.1007/s10803-013-1979-4

Cicchetti, D., and Cohen, D. J. (eds.). (2006). Developmental Psychopathology: Risk, Disorder, and Adaptation, 2nd Edn. New York, NY: John Wiley and Sons.

Cicchetti, D., and Rogosch, F. A. (1996). Equifinality and multifinality in developmental psychopathology. Dev. Psychopathol. 8, 597-600. doi: $10.1017 /$ S09545794400007318

Clauss, J. A., and Blackford, J. U. (2012). Behavioral inhibition and risk for developing social anxiety disorder: a meta-analytic study. J. Am. Acad. Child Adolesc. Psychiatry 51, 1066-1075. doi: 10.1016/j.jaac.2012.08.002

Cohan, S. L., Chavira, D. A., Shipon-Blum, E., Hitchcock, C., Roesch, S. C., and Stein, M. B. (2008). Refining the classification of children with selective mutism: a latent profile analysis. J. Clin. Child Adolesc. Psychol. 37, 770-784. doi: 10.1080/15374410802359759

Cohan, S. L., Price, J. M., and Stein, M. B. (2006). Suffering in silence: why a developmental psychopathology perspective on selective mutism is needed. J. Dev. Behav. Pediatr. 27, 341-355. doi: 10.1097/00004703-200608000-00011

Cornacchio, D., Furr, J. M., Sanchez, A. L., Hong, N., Feinberg, L. K., Tenenbaum, R., et al. (2019). Intensive group behavioral treatment for children with selective mutism: a preliminary randomized clinical trial. J. Consult. Clin. Psychol. 87, 720-733. doi: $10.1037 / \mathrm{ccp} 0000422$ and wrote the article. NM and LW were involved in the practical implementation of the study: they designed the web survey, collected, and processed the data. TO contributed to the theoretical foundation of the study and assisted in writing the article. All authors contributed to the article and approved the submitted version.

Diliberto, R., and Kearney, C. A. (2018). Latent class symptom profiles of selective mutism: identification and linkage to temperamental and social constructs. Child Psychiatry Hum. Dev. 49, 551-562. doi: 10.1007/s10578-017-0774-4

Driessen, J., Blom, J. D., Muris, P., Blashfield, R., and Molendijk, M. L. (2020). Anxiety in children with selective mutism: a meta-analysis. Child Psychiatry Hum. Dev. 51, 330-341. doi: 10.1007/s10578-019-00933-1

Edwards, S. L. (2007). Temperament and environmental risk factors contributing to anxiety symptoms in preschool-aged children (Ph.D. thesis). Macquarie University Australia, Sydney, NSW, Australia.

Edwards, S. L., Rapee, R. M., Kennedy, S. J., and Spence, S. H. (2010). The assessment of anxiety symptoms in preschool-aged children: the Revised Preschool Anxiety Scale. J. Clin. Child Adolesc. Psychol. 39, 400-409. doi: 10.1080/15374411003691701

Ersoy, M. (2019). Behavioral inhibition as an early marker of anxiety in children at risk for autism spectrum disorders (Ph.D. thesis). King's College London, London, United Kingdom.

Esroy, M., Charman, T., Pasco, G., Carr, E., Johnson, M. H., Jones, E. J. H., et al. (2020). Developmental paths to anxiety in an autism-enriched infant cohort: the role of temperamental reactivity and regulation. J. Autism Dev. Disord. doi: 10.1007/s10803-020-04734-7. [Epub ahead of print].

Garcia Coll, C., Kagan, J., and Reznick, J. S. (1984). Behavioral inhibition in young children. Child Dev. 55, 1005-1019. doi: 10.2307/1130152

Gensthaler, A., Dieter, J., Raisig, S., Hartmann, B., Ligges, M., Kaess, M., et al. (2020). Evaluation of a novel parent-rated scale for selective mutism. Assessment 27, 1007-1015. doi: 10.1177/1073191118787328

Gensthaler, A., Khalaf, S., Ligges, M., Kaess, M., Freitag, C. M., and Schwenck, C. (2016a). Selective mutism and temperament: the silence and behavioral inhibition to the unfamiliar. Eur. Child Adolesc. Psychiatry 25, 1113-1120. doi: 10.1007/s00787-016-0835-4

Gensthaler, A., Maichrowitz, V., Kaess, M., Ligges, M., Freitag, C. M., and Schwenck, C. (2016b). Selective mutism: the fraternal twin of childhood social phobia. Psychopathology 49, 95-107. doi: 10.1159/000444882

Goodman, L. A. (1961). Snowball sampling. Ann. Math. Stat. 32, 148-170. doi: $10.1214 /$ aoms/1177705148

Gudmundsdottir, H. R., Karlsson, T., and Aevarsdottir, T. (2019). The psychometric properties of the Icelandic version of the Preschool Anxiety scaleRevised. Nordic Psychol. 71, 218-232. doi: 10.1080/19012276.2019.1586571

Hudziak, J. J., Achenbach, T. A., Althoff, R. R., and Pine, D. S. (2007). A dimensional approach to developmental psychopathology. Int. J. Methods Psychiatry Res. 16, S16-S23. doi: 10.1002/mpr.217

Kagan, J. (1997). Temperament and reactions to unfamiliarity. Child Dev. 68, 139-143. doi: 10.1111/j.1467-8642.1997.tb01931.x

Karakaya, I., Sismanlar, S. G., Memik, N. C., Coskun, A., Agaoglu, B., and Yavuz, C. I. (2008). Selective mutism. A school-based cross-sectional study from Turkey. Eur. Child Adolesc. Psychiatry 17, 114-117. doi: 10.1007/s00787-007-0644-x

Klein, E. R., Ruiz, C. E., Morales, K., and Stanley, P. (2019). Variations in parent and teacher ratings of internalizing, externalizing, adaptive skills, and behavioral symptoms in children with selective mutism. Int. J. Environ. Res. Public Health 16:4070. doi: 10.3390/ijerph16214070

Kristensen, H. (2000). Selective mutism and comorbidity with developmental disorder/delay, anxiety disorder, and elimination disorder. J. Am. Acad. Child Adolesc. Psychiatry 39, 249-256. doi: 10.1097/00004583-200002000-0026

Krueger, R. F., and Piasecki, T. M. (2002). Toward a dimensional and psychometrically-informed approach to conceptualizing psychopathology. Behav. Res. Ther. 40, 485-499. doi: 10.1016/s0005-7967(02)0 0016-5 
Letamendi, A. M., Chavira, D. A., Hitchcock, C. A., Roesch, S. C., Shipon-Blum, E., and Stein, M. B. (2008). Selective Mutism Questionnaire: measurement structure and validity. J. Am. Acad. Child Adolesc. Psychiatry 47, 1197-1204. doi: 10.1097/CHIOb013e3181825a7b

Lodi-Smith, J., Rodgers, J. D., Cunningham, S. A., Lopata, C., and Thomeer, M. L. (2019). Meta-analysis of Big Five personality traits in autism spectrum disorders. Autism 23, 556-565. doi: 10.1177/1362361318766571

Macari, S. L., Koller, J., Campbell, D. J., and Chawarska, K. (2017). Temperamental markers in toddlers with autism spectrum disorder. J. Child Psychol. Psychiatry 58, 819-828. doi: 10.1111/jcpp. 12710

Magiati, I., Ong, C., Lim, X. Y., Tan, J. W., Ong, A. Y., Patrycia, F., et al. (2016). Anxiety symptoms in young peo- ple with autism spectrum disorder attending special schools: associations with gender, adaptive functioning and autism symptomatology. Autism 20, 306-320. doi: 10.1177/1362361315577519

Manassis, K. (2009). Silent suffering: understanding and treating children with selective mutism. Expert Rev. Neurother. 9, 235-243. doi: 10.1586/14737175.9.2.235

Manassis, K., Fung, D., Tannock, R., Sloman, L., Fiksenbaum, L., and McInnes, A. (2003). Characterizing selective mutism: is it more than social anxiety? Depress. Anxiety 18, 153-161. doi: 10.1002/da.10125

Manassis, K., Tannock, R., Garland, E. J., Minde, K., McInnes, A., and Clark, S. (2007). The sounds of silence: language, cognition, and anxiety in selective mutism. J. Am. Acad. Child Adolesc. Psychiatry 46, 1187-1195. doi: 10.1097/CHI.0b013e318076b7ab

Milic, M. I., Carl, T., and Rapee, R. M. (2020). Similarities and differences between young children with selective mutism and social anxiety disorder. Behav. Res. Ther. 133:103696. doi: 10.1016/j.brat.2020.103696

Mulligan, C. A. (2012). Selective mutism: identification of subtypes and influence on treatment (PCOM Psychology Dissertations), 210. Available online at: https:// digitalcommons.pcom.edu/psychology_dissertations/210

Muris, P., and Dietvorst, R. (2006). Underlying personality characteristics of behavioral inhibition in children. Child Psychiatry Hum. Dev. 36, 437-445. doi: 10.1007/s10578-006-0014-9

Muris, P., Hendriks, E., and Bot, S. (2016). Children of few words: relations among selective mutism, behavioral inhibition, and (social) anxiety symptoms in 3- to 6-year-olds. Child Psychiatry Hum. Dev. 47, 94-101. doi: $10.1007 / \mathrm{s} 10578-015-0547-\mathrm{x}$

Muris, P., and Ollendick, T. H. (2015). Children who are anxious in silence: a review on selective mutism, the new anxiety disorder in DSM-5. Clin. Child Fam. Psychol. Rev. 18, 151-169. doi: 10.1007/s10567-015-0181-y

Muris, P., and Ollendick, T. H. (2021a). Current diagnosis in the diagnosis and management of children with selective mutism. Psychol. Res. Behav. Manage. 14, 1-9. doi: 10.2147/PRBM.S274538

Muris, P., and Ollendick, T. H. (2021b). Selective mutism and its relations to social anxiety disorder and autism spectrum disorder. Clin. Child Fam. Psychol. Rev. 24, 294-325. doi: 10.1007/s10567-020-00342-0

Nowakowski, M. E., Tasker, S. L., Cunningham, C. E., McHolm, A. E., Edison, S., St Pierre, J., et al. (2011). Joint attention in parent-child dyads involving children with selective mutism: a comparison between anxious and typically developing children. Child Psychiatry Hum. Dev. 42, 78-92. doi: 10.1007/s10578-010-0208-Z

O'Brien, R. M. (2007). A caution regarding rules of thumb for variance inflation factors. Qual. Quant. 41, 673-690. doi: 10.1007/s11135-006-9018-6

Oerbeck, B., Overgaard, K. R., Bergman, R. L., Pripp, H., and Kristensen, H. (2020). The Selective Mutism Questionnaire: data from typically developing children and children with selective mutism. Clin. Child Psychol. Psychiatry 25, 754-765. doi: 10.1177/1359104520914695

Oerbeck, B., Stein, M. B., Pripp, A. H., and Kristensen, H. (2015). Selective mutism: follow-up study 1 year after end of treatment. Eur. Child Adolesc. Psychiatry 24, 757-766. doi: 10.1007/s00787-014-0620-1

Oerbeck, B., Stein, M. B., Wentzal-Larsen, T., Langsrud, O., and Kristensen, H. (2014). A randomized controlled trial of a home and school-based intervention for selective mutism: defocused communication and behavioral techniques. Child Adolesc. Ment. Health 19, 192-198. doi: 10.1111/camh. 12045

Ollendick, T. H., and Hirshfeld-Becker, D. R. (2002). The developmental psychopathology of social anxiety disorder. Biol. Psychiatry 51, 44-58. doi: 10.1016/S0006-3223(01)01305-1
Orgiles, M., Penosa, P., Fernandez-Martinez, I., Marzo, J. C., and Espada, J. P. (2018). Spanish validation of the Spence Preschool Anxiety Scale. Child Care Health Dev. 44, 753-758. doi: 10.1111/cch.12593

Pallathra, A. A., Calkins, M. E., Parish-Morris, J., Maddox, B. B., Perez, L. S., Miller, J., et al. (2018). Defining behavioral components of social functioning in adults with autism spectrum disorder as targets for treatment. Autism Res. 11, 488-502. doi: 10.1002/aur.1910

Perez-Edgar, K. E., and Guyer, A. E. (2014). Behavioral inhibition: temperament or prodrome? Curr. Behav. Neurosci. Rep. 1, 182-190. doi: 10.1007/s40473-014-0019-9

Plana-Ripoll, O., Pedersen, C. B., Holtz, Y., Benros, M. E., Dalsgaard, S. De Jonge, P., et al. (2019). Exploring comorbidity within mental disorders among a Danish national population. JAMA Psychiatry 76, 259-270. doi: 10.1001/jamapsychiatry.2018.3658

Poole, K. L., Cunningham, C. E., McHolm, A. E., and Schmidt, L. A. (2020). Distinguishing selective mutism and social anxiety in children: a multi-method study. Eur. Child Adolesc. Psychiatry. doi: 10.1007/s00787-020-01588-3. [Epub ahead of print].

Rapee, R. M., and Edwards, S. L. (2009). A selective intervention program for inhibited preschool-aged children of parents with an anxiety disorder: effects on current anxiety disorders and temperament. J. Am. Acad. Child Adolesc. Psychiatry 48, 602-609. doi: 10.1097/CHI.0b013e31819f6fa9

Rapee, R. M., Kennedy, S. J., Ingram, M., Edwards, S. L., and Sweeney, L. (2010). Altering the trajectory of anxiety in at-risk young children. Am. J. Psychiatry 167, 1518-1525. doi: 10.1176/appi.ajp.2010.09111619

Samyn, V., Roeyers, H., and Bijttebier, P. (2011). Effortful control in typically developing boys and in boys with ADHD or autism spectrum disorder. Res. Dev. Disabil. 32, 483-490. doi: 10.1016/j.ridd.2010. 12.038

Sharp, W. G., Sherman, C., and Gross, A. M. (2007). Selective mutism and anxiety: a review of the current conceptualization of the disorder. J. Anxiety Disord. 21, 568-579. doi: 10.1016/j.janxdis.2006.07.002

Spain, D., Sin, J., Linder, K. B., McMahon, J., and Happé, F. (2018). Social anxiety in autism spectrum disorder: a systematic review. Res. Autism Spectr. Disord. 52, 51-68. doi: 10.1016/j.rasd.2018.04.007

Spence, S. H., Rapee, R., McDonald, C., and Ingram, M. (2001). The structure of anxiety symptoms among preschoolers. Behav. Res. Ther. 39, 1293-1316. doi: 10.1016/s0005-7967(00)00098-x

Steffenburg, H., Steffenburg, S., Gillberg, C., and Billstedt, E. (2018). Autism spectrum disorder in children with selective mutism. Neuropsychiatr. Dis. Treat. 14, 1163-1169. doi: 10.2147/NDT.S154966

Stein, M. B., Yang, B. Z., Chavira, D. A., Hitchcock, C. A., Sung, S. C., Shipon-Blum, E., et al. (2011). A common genetic variant in the neurexin superfamily member CNTNAP2 is associated with increased risk for selective mutism and social anxiety-related traits. Biol. Psychiatry 69, 825-831. doi: 10.1016/j.biopsych.2010.11.008

Steinhausen, H. C., and Juzi, C. (1996). Elective mutism: an analysis of 100 cases. J. Am. Acad. Child Adolesc. Psychiatry 35, 606-614. doi: 10.1097/00004583-199605000-00015

Stuijfzand, S., and Dodd, H. F. (2017). Young children have social worries too: validation of a brief parent report measure of social worries in children aged 4-8 years. J. Anxiety Disord. 50, 87-93. doi: 10.1016/j.anxdis.2017.05.008

Teh, E. J., Chan, D. M., Tan, G. K. J., and Magiati, I. (2017). Continuity and change in, and child predictors of, caregiver reported anxiety symptoms in young people with autism spectrum disorder: a follow-up study. J. Autism Dev. Disord. 47, 3857-3871. doi: 10.1007/s10803-017-3136-y

Thom, R. P., Keary, C. J., Kramer, G., and Nowinski, L. A. (2020). Psychiatric assessment of social impairment across the lifespan. Harv. Rev. Psychiatry 28, 159-178. doi: 10.1097/HRP.000000000000257

Van Brakel, A. M. L., Muris, P., and Bögels, S. M. (2004). Relations between parent- and teacher-reported behavioral inhibition and behavioral observations of this temperamental trait. J. Clin. Child Adolesc. Psychol. 33, 579-589. doi: $10.1207 /$ s15374424jecp3303_15

Van der Ploeg, J. D., and Scholte, E. M. (2014). Autisme Spectrum Vragenlijst (ASV) [Autism Spectrum Questionnaire (ASQ)]. Houten: Bohn Stafleu Van Loghum.

Vecchio, J. L., and Kearney, C. A. (2005). Selective mutism in children: comparison to youth with and without anxiety disorders. J. Psychopathol. Behav. Assess. 27, 31-37. doi: 10.1007/s10862-005-3263-1 
Viana, A. G., Beidel, D. C., and Rabian, B. (2009). Selective mutism: a review and integration of the last 15 years. Clin. Psychol. Rev. 29, 57-67. doi: 10.1016/j.cpr.2008. 09.009

Vogel, F., Gensthaler, A., Stahl, J., and Schwenck, C. (2019). Fears and fear-related cognitions in children with selective mutism. Eur. Child Adolesc. Psychiatry 28, 1169-1181. doi: 10.1007/s00787-019-0 1281-0

Vreeke, L., Muris, P., Mayer, B., Huijding, J., Bos, A. E. R., Van der Veen, M., et al. (2012). The assessment of an inhibited, anxiety-prone temperament in a Dutch multi-ethnic population of preschool children. Eur. Child Adolesc. Psychiatry 21, 623-633. doi: 10.1007/s00787-0120299-0

Yeganeh, R., Beidel, D. C., and Turner, S. M. (2006). Selective mutism: more than social anxiety? Depress. Anxiety 23, 117-123. doi: 10.1002/da. 20139
Yeganeh, R., Beidel, D. C., Turner, S. M., Pina, A. A., and Silverman, W. K. (2003). Clinical distinctions between selective mutism and social phobia: an investigation of childhood psychopathology. J. Am. Acad. Child Adolesc. Psychiatry 42, 1069-1075. doi: 10.1097/01.CHI.0000070262.24125.23

Conflict of Interest: The authors declare that the research was conducted in the absence of any commercial or financial relationships that could be construed as a potential conflict of interest.

Copyright (C) 2021 Muris, Monait, Weijsters and Ollendick. This is an open-access article distributed under the terms of the Creative Commons Attribution License (CC $B Y)$. The use, distribution or reproduction in other forums is permitted, provided the original author(s) and the copyright owner(s) are credited and that the original publication in this journal is cited, in accordance with accepted academic practice. No use, distribution or reproduction is permitted which does not comply with these terms. 